\title{
Postoperative Velopharyngeal Closure Function Following Unified Velopharyngeal Plasty as Secondary Surgery for Cleft Palate: One- and Three-Year Follow-Up Results
}

\author{
Kenichi Kurita ${ }^{1}$, Yu Ito ${ }^{1}$, Atsushi Nakayama ${ }^{1}$, Atsushi Abe ${ }^{1}$, Kana Mukai ${ }^{1}$, \\ Kou Kawahara ${ }^{1}$, Michie Ito ${ }^{2}$, Miru Takami ${ }^{2}$, Michiko Shimooka ${ }^{3}$ \\ ${ }^{1}$ Department of Oral and Maxillofacial Surgery, School of Dentistry, Aichi-Gakuin University, Nagoya, Japan \\ ${ }^{2}$ Division of Speech Therapy, Aichi-Gakuin University Dental Hospital, Nagoya, Japan \\ ${ }^{3}$ Former Division of Speech Therapy, Aichi-Gakuin University Dental Hospital, Nagoya, Japan \\ Email: ken-kuri@dpc.aichi-gakuin.ac.jp
}

Received November 2, 2011; revised December 13, 2011; accepted December 25, 2011

\begin{abstract}
Velopharyngeal closure function following modified unified velopharyngeal plasty performed by a single surgeon was evaluated pre- and postoperatively in 14 children with cleft palate after cleft palate closure and no improvement by speech training. Subjects were evaluated for velopharyngeal closure function before and after surgery at 1 (short-term evaluation) and 3 (mid-term evaluation) years. Degree of hypernasality and degree of air leakage through the nose in the soft blowing test were each classified into three grades and each grade was given scores. Velopharyngeal closure function was classified according to the total score into four grades: "good", "fair", "slightly poor" and "poor". At the short-term evaluation, 11 patients (78.6\%) achieved an improvement to "good" or "fair" on the overall evaluation. More satisfactory results were obtained at the mid-term evaluation, with 14 patients (100\%) graded as "good" or "fair". Our results indicate that this modified unified velopharyngeal plasty effectively improves velopharyngeal insufficiency when performed as a secondary surgery for cleft palate in children.
\end{abstract}

Keywords: Velopharyngeal Insufficiency; Cleft Palate; Pharyngeal Flap Operation; Postoperative Speech Outcome; Velopharyngeal Closure Function

\section{Introduction}

Velopharyngeal closure function following cleft palate closure plays an important role in acquiring normal speech. Various techniques have been proposed for narrowing of the nasopharyngeal space and improvement of function. A number of studies have demonstrated the effectiveness of the pharyngeal flap operation for the treatment of velopharyngeal insufficiency.

Factors affecting postoperative velopharyngeal closure function following pharyngeal flap operation include preoperative velopharyngeal closure function, age at operation, the surgical procedure used, the width and attachment site of the pharyngeal flap, and postoperative speech training [1-5].

Our treatment algorithm for children with cleft palate is as follows. For newborns, a Hotz plate is made and placed at the first visit to improve suckling function and promote jaw development. Speech management is started at around 1 year of age, with two-stage palatoplasty [6] applied for children with cleft palate at our department. In consideration of the child's "time to start speaking", the initial palatoplasty is performed at 1.5 to 2 years using the mucosal flap technique proposed by Perko [7]. A speech plate is used to close a cleft of the hard palate. Speech is assessed by speech therapists at 4 years. Thereafter, regular speech training and adjustments of the speech plate and the speech aid are made. Pharyngeal flap operation is carried out for those patients with velopharyngeal insufficiency which failed to show improvement over time and could not be maintained by conservative treatment. Cleft of the hard palate is closed with alveolar bone grafting at 8 to 10 years.

Among a number of techniques that have been developed for pharyngeal flap operation, including costal cartilage grafting [8] and the folded pharyngeal flap technique proposed by Isshiki et al. [9-12], the superior flap technique is most commonly used. The surgical technique used in our hospital is a modified form of unified velopharyngeal plasty proposed by Tachimura et al. [13-15] (hereinafter, modified UVP), which is classified as a superior flap technique based on the shape of the flap formed. The surgical outcomes of pharyngeal flap opera- 
tion vary substantially among surgeons and thus cannot be simply compared [10]. Thus, the present study included patients who had undergone modified UVP by the same surgeon and evaluated the velopharyngeal closure function of these patients at 1 and 3 years postoperation.

\section{Methods}

\subsection{Subjects}

The study subjects were 14 children with cleft palate who presented to the clinic of the Department of Oral and Maxillofacial Surgery, Aichi-Gakuin University Dental Hospital and were diagnosed with velopharyngeal insufficiency, for which speech training didn't provide no improvement because the lateral cephalometric radiograph showed the soft palate was too short to reach the posterior wall of the pharynx. They subsequently underwent modified UVP performed by the same surgeon as secondary surgery and who were available for follow-up speech assessments for more than 3 years postoperation.

Orifices for the purpose of ventilation were created bilaterally with a diameter of about $5 \mathrm{~mm}$ [11]. For a cleft of the hard palate, the mucosal flap technique was also used to minimize development of the maxillary and palatal bones [15]. All of the 14 patients had undergone preoperative speech training at our or other hospitals and had been considered unlikely to make improvement by conservative treatment alone. Exclusion criteria were congenital velopharyngeal insufficiency, and mental retardiation.

\subsection{Evaluation of Velopharyngeal Closure Function}

Postoperative evaluation consisted of the short-term evaluation performed at 1 year postoperation, by which time the condition was assumed to be stabilized [16-18], and the mid-term evaluation performed at 3 years postoperation. For those patients who were unavailable at the 3year evaluation, data were obtained at the visit closest to 3 years were used for evaluation.

The evaluation method used in this study was based on Methods for Testing Closure Function issued by the Cleft Palate and Speech Subcommittee of The Japan Society of Logopedics and Phoniatrics [19]. A speech therapist performed the auditory evaluation of vocal language by classifying the degree of hypernasality into 3 grades: "negative", "slightly positive" and "positive". Each grade was then assigned a score of 0,1 or 2 points, respectively (Table 1).

In the soft blowing test, each subject was asked to blow through a straw into a cup and the maximum values of misting measured on the surface of a nasal breath mirror on each side were classified into 3 grades. A score of 0 point was given for values $\leq 1,1$ point for values $>1$ and $\leq 2$, and 2 points for values $>2$ (Table 1$)$.

Overall evaluation was based on the total scores from both tests; a total score of 0 point was defined as "good", 1 point as "fair", 2 points as "slightly poor" and 3 and 4 points as "poor" (Table 2).

\section{Results}

\subsection{Subjects (Table 3)}

Six of the 14 children had been treated from birth at our department by our algorithm and the cleft palate was closed in two-stage in the 4 children. Other 8 children were referred from other clinics to our department during the treatments. The average age at pharyngeal flap operation was 8 years 6 months (range, 3 years 8 months to 18 years 3 months; median, 7 years 0 months).

\subsection{Evaluation of Velopharyngeal Closure Function (Table 3)}

At the preoperative evaluation of hypernasality among 14 patients, 3 patients $(21.4 \%)$ were classified as "slightly positive" and 11 patients $(78.6 \%)$ as "positive". At the shortterm evaluation, 10 patients $(71.4 \%)$ were classified as "negative" and 4 patients (28.6\%) as "slightly positive". The 4 pa- tients with a short-term hypernasality evaluation of "sli- ghtly positive" were classified as "good" on evaluation of single sound pronunciation, but were clas-

Table 1. Rating and scoring for each test.

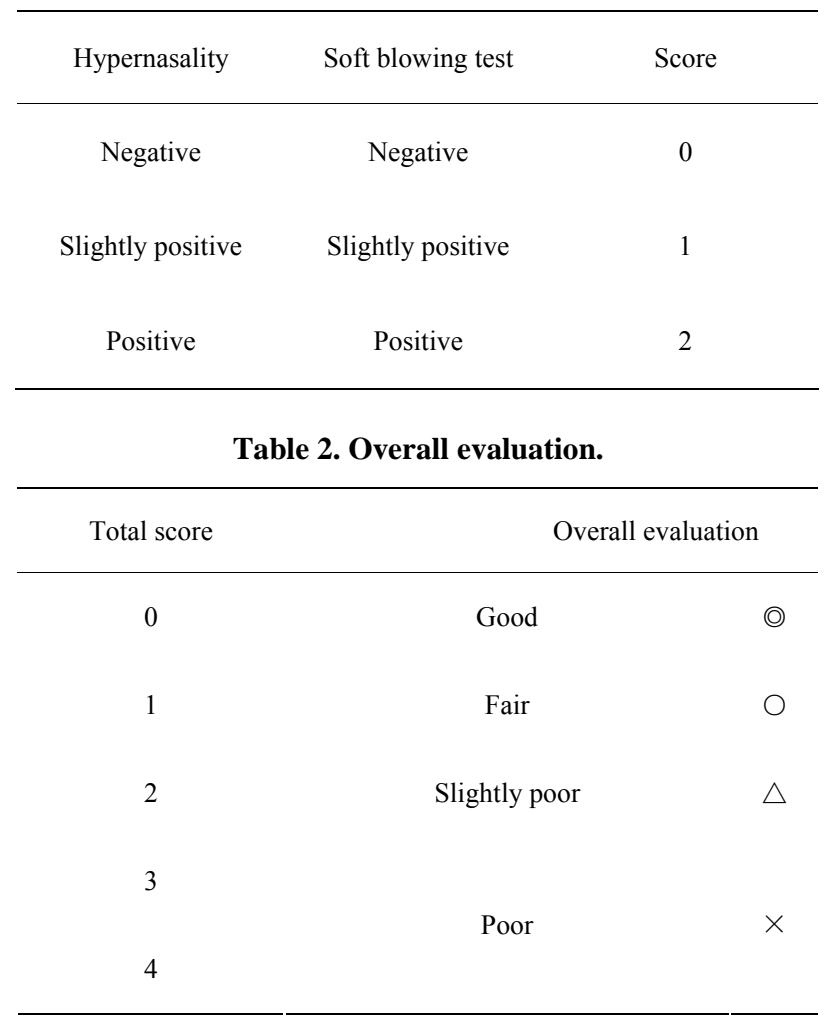


Table 3. Time course of velopharyngeal function in each patient.

\begin{tabular}{|c|c|c|c|c|c|c|c|c|c|}
\hline \multirow{2}{*}{ Patient No. } & \multicolumn{2}{|c|}{ Age at operation } & \multirow{2}{*}{ Cleft type } & \multirow{2}{*}{ Past treatment } & \multirow{2}{*}{ Palatal closure } & \multirow{2}{*}{$\begin{array}{c}\text { Hypernasality } \\
\mathrm{Pre} \rightarrow \text { Short } \rightarrow \text { Mid }\end{array}$} & \multirow{2}{*}{$\frac{\text { Blowing test }}{\mathrm{Pre} \rightarrow \text { Short } \rightarrow \text { Mid }}$} & \multirow{2}{*}{$\frac{\text { Total score }}{\text { Pre } \rightarrow \text { Short } \rightarrow \text { Mid }}$} & \multirow{2}{*}{$\begin{array}{l}\text { Overall evaluation } \\
\mathrm{Pre} \rightarrow \mathrm{Short} \rightarrow \mathrm{Mid}\end{array}$} \\
\hline & years & months & & & & & & & \\
\hline 1 & 8 & 1 & UCLP & $x$ & 1 & $1 \rightarrow 0 \rightarrow 0$ & $2 \rightarrow 0 \rightarrow 0$ & $3 \rightarrow 0 \rightarrow 0$ & $x \rightarrow(0 \rightarrow 0$ \\
\hline 2 & 11 & 11 & $\mathrm{CP}$ & 0 & 1 & $2 \rightarrow 0 \rightarrow 0$ & $2 \rightarrow 0 \rightarrow 0$ & $4 \rightarrow 0 \rightarrow 0$ & $x \rightarrow(0 \rightarrow(0)$ \\
\hline 3 & 4 & 8 & $\mathrm{CP}$ & 0 & 1 & $2 \rightarrow 0 \rightarrow 0$ & $1 \rightarrow 0 \rightarrow 0$ & $3 \rightarrow 0 \rightarrow 0$ & $x \rightarrow(0 \rightarrow(0)$ \\
\hline 4 & 5 & 10 & UCLP & $x$ & 1 & $2 \rightarrow 0 \rightarrow 0$ & $1 \rightarrow 0 \rightarrow 0$ & $3 \rightarrow 0 \rightarrow 0$ & $x \rightarrow @ \rightarrow(0$ \\
\hline 5 & 18 & 3 & UCLP & $x$ & 1 & $2 \rightarrow 1 \rightarrow 0$ & $1 \rightarrow 0 \rightarrow 0$ & $3 \rightarrow 1 \rightarrow 0$ & $\times \rightarrow O \rightarrow(0$ \\
\hline 6 & 4 & 11 & UCLP & $\mathrm{O}$ & 2 & $2 \rightarrow 0 \rightarrow 0$ & $1 \rightarrow 0 \rightarrow 0$ & $3 \rightarrow 0 \rightarrow 0$ & $x \rightarrow(0 \rightarrow(0$ \\
\hline 7 & 5 & 5 & $\mathrm{CP}$ & $x$ & 1 & $2 \rightarrow 0 \rightarrow 0$ & $1 \rightarrow 0 \rightarrow 0$ & $3 \rightarrow 0 \rightarrow 0$ & $x \rightarrow(0 \rightarrow(0$ \\
\hline 8 & 11 & 6 & BCLP & $x$ & 1 & $2 \rightarrow 0 \rightarrow 0$ & $1 \rightarrow 1 \rightarrow 0$ & $3 \rightarrow 1 \rightarrow 0$ & $\times \rightarrow O \rightarrow(0$ \\
\hline 9 & 10 & 2 & $\mathrm{CP}$ & $x$ & 1 & $1 \rightarrow 0 \rightarrow 0$ & $1 \rightarrow 0 \rightarrow 0$ & $2 \rightarrow 0 \rightarrow 0$ & $\triangle \rightarrow \bigcirc \rightarrow(0$ \\
\hline 10 & 3 & 8 & UCLP & $\mathrm{O}$ & 2 & $2 \rightarrow 1 \rightarrow 0$ & $2 \rightarrow 1 \rightarrow 1$ & $4 \rightarrow 2 \rightarrow 1$ & $x \rightarrow \triangle \rightarrow 0$ \\
\hline 11 & 5 & 10 & $\mathrm{CP}$ & $x$ & 1 & $2 \rightarrow 0 \rightarrow 0$ & $2 \rightarrow 0 \rightarrow 0$ & $4 \rightarrow 0 \rightarrow 0$ & $x \rightarrow(0 \rightarrow 0$ \\
\hline 12 & 17 & 3 & UCLP & $x$ & 1 & $2 \rightarrow 1 \rightarrow 0$ & $2 \rightarrow 1 \rightarrow 0$ & $4 \rightarrow 2 \rightarrow 0$ & $x \rightarrow \triangle \rightarrow 0$ \\
\hline 13 & 4 & 9 & UCLP & $\mathrm{O}$ & 2 & $1 \rightarrow 0 \rightarrow 0$ & $2 \rightarrow 0 \rightarrow 1$ & $3 \rightarrow 0 \rightarrow 1$ & $x \rightarrow(0 \rightarrow 0$ \\
\hline 14 & 8 & 6 & UCLP & $\bigcirc$ & 2 & $2 \rightarrow 1 \rightarrow 0$ & $2 \rightarrow 1 \rightarrow 0$ & $4 \rightarrow 2 \rightarrow 0$ & $\times \rightarrow \triangle \rightarrow 0$ \\
\hline
\end{tabular}

BCLP, bilateral cleft lip and palate; UCLP, unilateral cleft lip and palate; CP, cleft palate; Past treatment: $\bigcirc$; treatmented at our department from birth $\times$; referned from other clinics; Palatal closure: 1: one-stage, 2: two-stage.

sified as "slightly positive" with regard to hypernasality during conversation. At the mid-term evaluation, all the 14 patients (100\%) were classified as "negative" (Figure 1).

The results of the preoperative soft blowing test among the 14 patients were "slightly positive" in 7 patients $(50.0 \%)$ and "positive" in 7 patients $(50.0 \%)$. The test results at the short-term evaluation were "negative" in 10 patients $(71.4 \%)$ and "slightly positive" in 4 patients $(28.6 \%)$, and those at the mid-term evaluation were "negative" in 12 patients $(85.7 \%)$ and "slightly positive" in 2 patients (14.3\%) (Figure 2).

The overall evaluation of preoperative velopharyngeal closure function was "poor" in 13 patients $(92.9 \%)$ and "slightly poor" in 1 patient (7.1\%). At the short-term evaluation, 9 patients $(64.3 \%)$ were rated as "good", 2 patients $(14.3 \%)$ as "fair", and 3 patients $(21.4 \%)$ as "slightly poor". At the mid-term evaluation, 12 patients $(85.7 \%)$ were rated as "good" and 2 patients (14.3\%) as "fair" (Figure 3).

In terms of the effect of surgery, 11 patients $(78.6 \%)$ showed "much improvement (improvement of two grades or more)", such as improvement from "poor" at the pre operative evaluation to "fair" at the short-term evaluation, and 3 patients (21.4\%) showed "improvement (improvement of one grade)", such as improvement from "poor" to "slightly poor". At the mid-term evaluation, compared with the short-term evaluation, 8 patients $(57.1 \%)$ remained at the "good" rating, 5 patients $(35.7 \%)$ showed "improvement", such as improvement from "slightly poor" or "fair" to "good" or " fair", and 1 patient $(7.1 \%)$ showed worsening of overall evaluation. All patients showed improvement at the postoperative

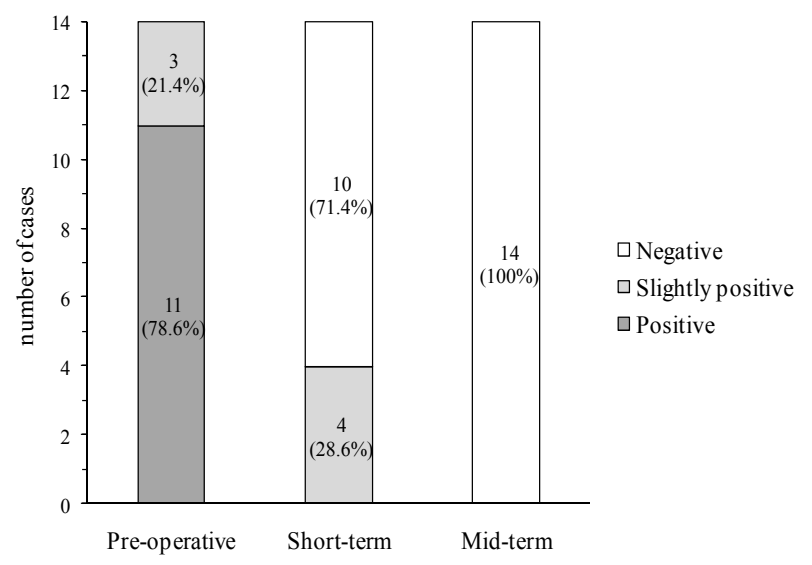

Figure 1. Evaluation of hypernasality. 


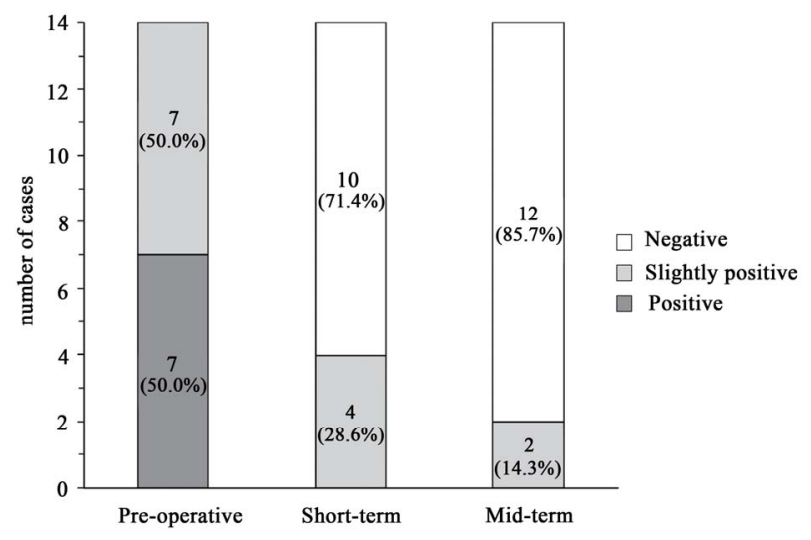

Figure 2. Soft blowing test results.

evaluation compared with the preoperative evaluation.

Three of the 14 patients $(21.4 \%)$ presented mild hyponasality at the short-term evaluation. None of the 3 patients required removal of the pharyngeal flap or presented wi- th sleep apnea syndrome. All the 3 patients showed an improvement in hyponasality at the mid-term evaluation.

Postoperative visual motility assessment of the soft palate following pharyngeal flap operation revealed no findings suggestive of motility disorder or unilateral motor paralysis in any of the patients.

\section{Discussion}

In the present study, the percentage of the patients rated as "good" or "fair" on the overall evaluation of postoperative velopharyngeal closure function was $78.6 \%$ at the short-term evaluation and $100 \%$ at the mid-term evaluation. These results are comparable to those reported in previous studies, in which patients with good function accounted for $70 \%-100 \%$ and those with mild insufficiency accounted for $17 \%$ - 30\% of all patients $[16,17$, 20-25]. Factors contributing to the favorable postoperative outcomes achieved in the present study include adequate backward displacement of the soft palate, appropriate width, length and location of the pharyngeal flap to immobilize the soft palate, minimal postoperative contraction of the transplanted flap, and absence of complications resulting from minimal intraoperative bleeding, with a mean operative bleeding of around $80 \mathrm{~g}$ as reported by Tachimura [13-15]. In addition, since only very small areas of the raw surfaces of the soft palate and pharynx are exposed in modified UVP, the resulting scar tissue can be minimized and the mobility of the transplanted flap and surrounding tissues can be preserved at the maximum level.

We were not sure whether anterior part of the hard pate should be first closed prior to velopharyngeal plasty. Out of 4 patients (patient \#6, \#10, \#13 and \#14) with

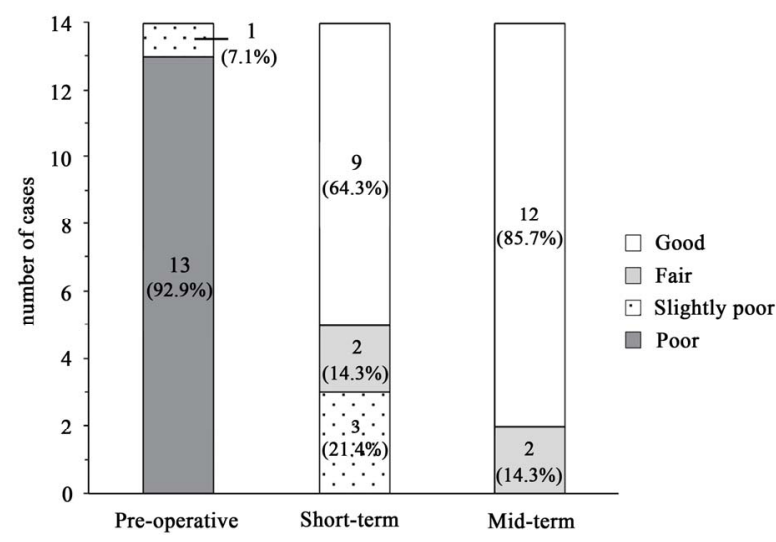

Figure 3. Overall evaluation.

unilateral cleft lip and palate under two-stage palatal closure algorithm, 2 patients (patient \#10 and \#14) presented with "slightly poor" velopharyngeal closure function at the short-term evaluation. These patients had an unclosed cleft in the anterior part of the hard palate and had a speech plate placed at the time of the short-term postoperative evaluation. These patients underwent closure of a residual cleft of the hard palate and alveolar cleft at 10 years and subsequently achieved improvement in velopharyngeal closure function. Therefore, the reason for the "slightly poor" rating on the velopharyngeal closure function in these patients may be because the cleft in the anterior part of the hard palate was not sufficiently covered by the speech plate and the resulting air leakage through the nose affected the velopharyngeal closure function. Furthermore, only 1 patient (patient \#13) showed worsening of velopharyngeal closure function from "good" at the short-term evaluation to "fair" at the mid-term evaluation. This patient was also undergoing two-stage palatoplasty [2] and was under orthodontic treatment at the mid-term evaluation. This patient underwent closure of a residual cleft of the hard palate and alveolar cleft at 10 years and also subsequently achieved improvement in velopharyngeal closure function after the mid-term evaluation. The reason for the worsening of velopharyngeal closure function may therefore be due to the incomplete closure of the residual cleft in the anterior part of the hard palate with an ill-fitting speech plate. Thus, it is recommended that hard cleft palate is first closed prior to velopharyngeal plasty for children with velopharyngeal insufficiency in the two-stage palatoplasty.

Okazaki et al. [17] identified older age at operation as a risk factor for postoperative hypernasality. One of three patients (patient \#12) with "slightly poor" velopharyngeal closure function at the short-term evaluation was 17 years 3 months. This patient, even after pharyngeal flap operation and subsequent improvement in velopharyngeal closure function, would unconsciously speak using old articulation patterns learned and used before surgery, 
and thus required a long time to learn and adopt new articulation patterns. Patient \#5 underwent surgery at the age of 18 years 3 months. This patient also achieved improvement to "fair" at the short-term evaluation, but had a hypernasality evaluation of "slightly positive". However, the overall evaluation at mid-term was good in these high teens. Therefore, this modified UVP could improve velopharyngeal closure function of high teens until the midterm evaluation.

On the other hand, 10 of the 12 patients who underwent surgery at less than 12 years achieved improvement at the short-term evaluation (improvement rate: $83.3 \%$ ). The 2 patients without improvement were undergoing twostage palatoplasty for cleft palate, as mentioned above. Given that these patients also achieved improvement following closure of a residual cleft of the hard palate, the present results demonstrate the effectiveness of performing surgery at less than 10 years.

Pharyngeal flap operation in children at younger ages is associated with the risk of recurrence of velopharyngeal insufficiency as a result of the physiological growth of facial bones during later childhood [26]. Repeated surgeries on the hard palate may also affect jaw development. With regard to this issue, the palatoplasty and pharyngeal flap operation techniques employed at our hospital involve the application of the mucosal flap technique to the hard palate and thus is likely to have minimal impact on jaw development. Future studies must consider recurrence of velopharyngeal insufficiency and the longer-term impact of the surgical techniques on jaw development.

The evaluation method used in the present study was based on Velopharyngeal Methods for Testing Closure Function issued by the Cleft Palate and Speech Subcommittee of The Japan Society of Logopedics and Phoniatrics [19]. Other reported methods include measuring acoustic energy with a nasometer [27-29], measuring the size of the soft palate on lateral cephalometric radiographs [29] and use of a posterior rhinoscope [30]. These methods were not employed in the present study because they require special measuring instruments and a well-equipped recording room, and are also associated with the problems of radiation exposure. Future studies should introduce digitalized voice assessment by mechanical measurement and seek to develop new evaluation methods utilizing voice data gathered for individual patients.

Our results demonstrated that modified UVP for postoperative velopharyngeal insufficiency due to cleft palate provided a high improvement rate $(78.6 \%)$ at the shortterm evaluation. Subsequent closure of a residual cleft and patient growth and development resulted in an improvement to "fair" or better at the mid-term evaluation in all patients, again demonstrating the effectiveness and usefulness of the surgical technique. Although perform- ing surgery in the late teens resulted in a slight delay in postoperative improvement in velopharyngeal closure function, performing it at younger ages was not associated with postoperative complications or recurrence at the midterm evaluation. These findings indicate the appropriateness of applying modified UVP to children from younger to high teens with postoperative velopharyngeal insufficiency following palatoplasty which is unlikely to be improved by non-invasive treatment. Further studies are warranted to examine the longer-term clinical course including recurrence of velopharyngeal insufficiency.

\section{Conclusion}

Fourteen children who had undergone palatoplasty and were diagnosed as having velopharyngeal insufficiency by postoperative speech assessment underwent modified UVP by the same surgeon. Short-term and mid-term evaluations were performed at 1 and 3 years postoperation. The degree of hypernasality and the soft blowing test were evaluated and results were converted into scores. According to the total scores for both tests, velopharyngeal closure function was classified into four grades: "good", "fair", "slightly poor" and "poor". At the short-term evaluation, 11 of the 14 patients $(78.6 \%)$ were classified as "good" or "fair". At 3 years postoperation, all of the 14 patients $(100 \%)$ were classified as "good" or "fair". Our results indicate that this modified UVP is effective to improve velopharyngeal insufficiency as secondary surgery for cleft palate in children. It is recommended that hard palate closure is first carried out prior to velopharyngeal plasty for children with velopharyngeal insufficiency in the two-stage palatoplasty.

\section{Acknowledgements}

The authors wish to express their deep appreciation to the children with cleft palate and their parents who have given their time and effort to this research.

\section{REFERENCES}

[1] K. R. Bzoch, "Clinical Studies of the Efficacy of Speech Appliances Compared to Pharyngeal Flap Surgery," Cleft Palate Journal, Vol. 35, 1964, pp. 275-286.

[2] M. Hamlen, "Speech Changes after Pharyngeal Flap Surgery," Plastic and Reconstructive Surgery, Vol. 46, No. 5, 1970, pp. 437-444. doi:10.1097/00006534-197011000-00004

[3] H. L. Morris and D. C. Spriestersbach, "The Pharyngeal Flap as a Speech Mechanism," Plastic and Reconstructive Surgery, Vol. 39, No. 1, 1967, pp. 66-70. doi:10.1097/00006534-196701000-00007

[4] J. Q. Owsley Jr., B. J. Creech and H. H. Dedo, "Poor Speech Following the Pharyngeal Flap Operation; Etiology and Treatment," Cleft Palate Journal, Vol. 9, 1972, pp. 312-318. 
[5] A. Taen, N. Tsutsumi, Y. Ogata and Y. Kakihara, "Studies on the Effects of the Pharyngeal Flap Operation on the Soft Palate Insufficiency [in Japanese]," Journal of the Kyushu Dental Society, Vol. 24, No. 6, 1971, pp. 811820.

[6] K. Kurita, A. Nakayama, C. Kurata, M. Shimizu, K. Mukai, M. Isobe, et al., "Two-Stage Closure of Cleft Palate : Our Protocol [in Japanese]," Journal of Japanese Cleft Palate Association, Vol. 32, No. 1, 2007, pp. 17-23.

[7] M. A. Perko, "Two-Stage Closure of Cleft Palate (Progress Report)," Journal of Maxillofacial Surgery, Vol. 7, No. 1, 1979, pp. 46-80.

[8] N. Kimura, K. Nakamura and N. Kido, "A Costal Cartilage Graft of the Posterior Pharynx in Velopharyngeal Incompetence Cases [in Japanese]," Japanese Journal of Plastic Surgery, Vol. 47, No. 6, 2004, pp. 593-600.

[9] N. Isshiki, "Folded Pharyngeal Flap [in Japanese]," Pract Otologica, Vol. 69, 1976, pp. 211-214.

[10] N. Isshiki, "Congenital Velopharyngeal Insufficiency and Rhinophonia: Medical Practice for Rhinophonia [in Japanese]," Vol. 17, JOHNS, Tokyo-igakusha, Tokyo, 2001, pp. 1147-1151.

[11] N. Isshiki, I. Honjo and H. Maeda, "Operations for Velopharyngeal Insufficiency,” Practica Otologica, Vol. 62, 1969, pp. 1113-1120.

[12] N. Isshiki and M. Morimoto, "A New Folded Pharyngeal Flap. Preliminary Report," Plastic and Reconstructive Surgery, Vol. 55, No. 4, 1975, pp. 461-465.

[13] T. Tachimura, H. Hara, T. Wada, S. Iida, M. Kogo and T. Matsuya, "Unified Velopharyngoplasty for the Management of Recurrence of Velopharyngeal Incompetence after Pharyngeal Flap Surgery [in Japanese]," Japanese Journal of Oral and Maxillofacial Surgery, Vol. 42, No. 3, 1996, pp. 320-322. doi:10.5794/jijoms.42.320

[14] T. Tachimura, H. Hara, T. Wada, S. Iida, M. Kogo and T. Matsuya, "Unified Velopharyngoplasty for the Management of Recurrence of Velopharyngeal Incompetence after Pharyngeal Flap Surgery [in Japanese]," Japanese Journal of Oral and Maxillofacial Surgery, Vol. 42, No. 3, 1996, pp. 320-322. doi:10.5794/jioms.42.320

[15] T. Tachimura and T. Wada, "A Modified Procedure in Pharyngeal Flap Operation with Palatal Mucosal Flap Technique and with Fibrin Sealant (Tisseel) [in Japanese]," Journal of Japanese Cleft Palate Association, Vol. 14, No. 3, 1989, pp. 391-401.

[16] H. Hoshikawa, R. Goto, M. Karaki, K. Miyabe and N. Mori, "A Case Report of Pharyngeal Flap Operation in Patients with Velopharyngeal Insufficiency [in Japanese]," Journal of Otolaryngology of Japan, Vol. 106, No. 6, 2003, pp. 700-704.

[17] K. Okazaki, M. Kato, T. Onizuka, N. Sumiya, H. Gyotoku and T. Sambe, "Speech Outcome Following Pharyngeal Flap Operation [in Japanese]," Japanese Journal of Plastic Surgery, Vol. 31, No. 3, 1988, pp. 202-208.

[18] T. Sonoda, "Study of Velopharyngeal Movement in Cleft Palate Patients Following Pharyngeal Flap Surgery [in Japanese]," Journal of Japanese Cleft Palate Association, Vol. 26, No. 1, 2001, pp. 68-87.
[19] A. Ohira, K. Okazaki, N. Ainoda, M. kato, F. Tanokuchi, T. Fukuda, et al., "Methods for Testing Velopharyngeal Closure Function [in Japanese]," Japanese Journal of Logoped Phoniatria, Vol. 34, No. 3, 1993, pp. 298-304. doi:10.5112/jilp.34.298

[20] M. Hiramoto and Y. Fujiwara, "Evaluation of the Pharyngeal Flap and Re-Extension Techniques Based on Speech Outcome [in Japanese]," Japanese Journal of Plastic Surgery, Vol. 40, No. 6, 1997, pp. 547-552.

[21] T. Horibe, K. Naito, K. Takeuchi, S. Saito, H. Asahi, C. Ito, et al., "Veropharyngeal Competence and General Health Conditions after Pharyngeal Flap Construction in Cleft Palete Children [in Japanese]," Pediatric Otorhinolaryngology Japan, Vol. 25, No. 2, 2004, pp. 56-59.

[22] M. Kawano, N. Isshiki, Y. Harita, F. Tanokuchi, M. Yamada, K. Kuniyoshi, et al., "Treatment and Result of Slight Velopharyngeal Incompetence," Studia phonologica, Vol. 17, 1983, pp. 15-26.

[23] T. Takato, M. Itoh, M. Kamei, K. Uchiyama, I. Kitano and H. Takeda, "Evaluation of Velopharyngeal Function after Pharyngeal Flap Surgery [in Japanese]," Journal of Japanese Society of Plastic Reconstruction Surgery, Vol. 8, No. 5, 1988, pp. 435-443.

[24] T. Takato, S. Park, I. Kitano, K. Kato, T. Komori, T. Susami, et al., "Results of Velopharyngeal Function and Compliocations after Pharyngeal Flap Surgery in Preschool Children [in Japanese]," Journal of Japanese Cleft Palate Association, Vol. 19, No. 2, 1994, pp. 57-65.

[25] M. Miura, W. Yoko and M. Hirano, "Velopharyngeal Function Following Pharyngeal Flap Operation (Hirano Method) [in Japanese]," Japanese Journal of Logoped Phoniatria, Vol. 25, No. 4, 1984, pp. 285-294. doi: $10.5112 / \mathrm{jilp} .25 .285$

[26] D. R. Van Demark and M. A. Hardin, "Longitudinal Evaluation of Articulation and Velopharyngeal Competence of Patients with Pharyngeal Flaps," Cleft Palate Journal, Vol. 22, No. 3, 1985, pp. 163-172.

[27] S. Kondoh, S. Yuzuriha, D. Fukasawa, Y. Mishima, Y. Sakaguchi, S. Ohike, et al., "Objective Instrumental Evaluation of Velopharyngeal Insufficiency Using Nasometer and Nasalance Scores [in Japanese]," Journal of Japanese Society of Craniomaxillofacial Surgery, Vol. 19, No. 2, 2003, pp. 101-109.

[28] Y. Ogata, N. Nakamura, Y. Kubota, M. Sasaguri, R. Kikuta, K. Shirasuna, et al., "Nasometer Test for Analysis on Velopharyngeal Function of Patients with Cleft Palate : Objective Criteria for Velopharyngeal Function [in Japanese]," Journal of Japanese Cleft Palate Association, Vol. 28, No. 1, 2003, pp. 9-19.

[29] T. Uchiyama, Y. Motohashi, K. Yamamoto, H. Watanabe, H. Koeda, Y. Nakano, et al., "Methods of Assessing Velopharyngeal Function in Cleft Palate Patients [in Japanese]," Journal of Tokyo Dental College Society, Vol. 99, No. 8, 1999, pp. 641-656.

[30] A. Yuge, Y. Yamazaki, K. Suzuki, N. Nakakita, S. Okabe and K. Sezaki, "Evaluation of Velopharyngeal Closure Function with a Posterior Rhinoscope [in Japanese]," Journal of Japanese Cleft Palate Association, Vol. 2, No. 2, 2004, p. 220. 